\title{
Mass Generations in Two-Dimensional Hierarchical Heisenberg Model of Migdal-Kadanoff Type
}

\author{
Keiichi R. Ito
}

Department of Mathematics, College of Liberal Arts, Kyoto University, Kyoto, 606 Japan

\begin{abstract}
Finiteness of correlation length in the 2D Heisenberg model is established within the Migdal-Kadanoff approximate renormalization recursion formulas.
\end{abstract}

\section{Introduction}

Though it is well known that the two-dimensional (2D) $O(N)$-invariant statistical mechanical models $(N \geqq 2)$ with short range interactions do not exhibit any longrange orders $[4,18]$, we still do not have a rigorous proof for our longstanding conjecture that the $2 \mathrm{D} O(N)$-invariant statistical mechanical models would always have non-zero masses for all inverse temperatures $\beta \geqq 0$, provided $N$ is larger than or equal to 3 . This problem is rather similar to another longstanding problem of quark confinements in $4 \mathrm{D}$ non-Abelian lattice gauge theories $[19,1,5]$. These problems are probably solved only by hard analysis like real space renormalization group methods $[6,14,20]$. Unfortunately, the real space renormalization group methods are sometimes very complicated. Thus some approximate renormalization group methods were invented [13-15] and there have been some analytic studies $[2,3,6,8,9]$ though it is not easy (almost impossible) to know to what extent the approximate renormalization group methods are precise compared with the real systems. The present author recently proved that $4 \mathrm{D}$ nonAbelian lattice gauge theories always exhibit quark confinements (in the sense of Wilson) within the Migdal-Kadanoff approximate renormalization group methods that are believed to be rather accurate as the first approximation $[13,15$, $10,11]$, see also $[16,17]$.

In the case of the Heisenberg model in which $s_{x}$, the spin variable at the lattice point $x \in Z^{2}$, takes its values on $S^{2}=\left\{\left(s^{1}, s^{2}, s^{3}\right) \in R^{3} ;\|s\|=1\right\}$, the methods developed in [11] (see also [12]) do not work, and we need new tricks. These new tricks together with the methods in [11] enable us to establish:

Theorem. The 2D Heisenberg model is always in the massive phase within the Migdal-Kadanoff approximate renormalization group methods. 
Since we do not know the accuracy of the present approximate methods, this theorem may have to be taken with a grain of salt, see the final section of this paper. However, the analysis developed here shares some of the important features of the real space renormalization group methods, and this conclusion is very encouraging.

\section{Models}

The Migdal-Kadanoff renormalization group methods, first proposed by Migdal [15] and later reexamined by Kadanoff [13] by his bond moving method, hold exactly on hierarchical lattices embedded in $Z^{D}(D=2$ in the present case). See Fig. 1 which is borrowed from ref. 3 and drawn for $D=2, r=2$ [see Eq. (1a)]. Or equivalently these hierarchical lattices are made by steps shown in Fig. 2-M and Fig. 2-K, respectively. See also refs. $[8,9]$ for the construction of hierarchical lattices.

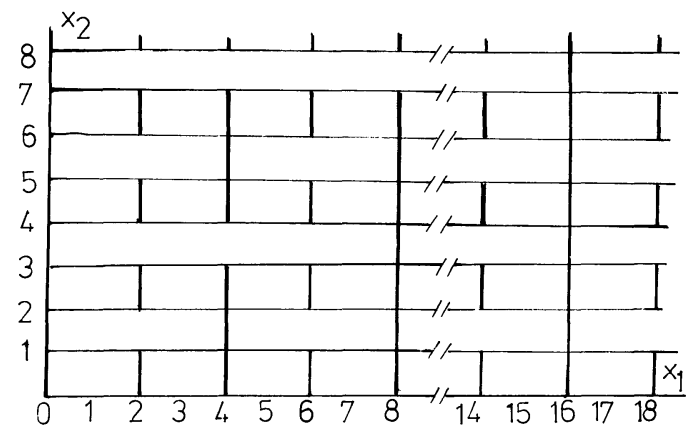

Fig. 1. The construction of a hierarchical lattice in which Migdal's recursion formula holds exactly. This corresponds to $r=2$ and $D=2$. For horizontal bonds $\left\langle x, x+e_{1}\right\rangle, e_{1}=(1,0)$, there correspond the standard actions $\exp \left[\beta\left(s_{x} \cdot s_{x+e_{1}}-1\right)\right]$, and for vertical bonds $\left\langle x, x+e_{2}\right\rangle$, $e_{2}=(0,1)$, we set $\beta$ in $\exp \left[\beta\left(s_{x} \cdot s_{x+e_{2}}-1\right)\right] \infty$ (then $s_{x}=s_{x+e_{2}}$ ) or 0 (then $s_{x}$ and $s_{x+e_{2}}$ are independent) depending on where they are. In this figure, $\beta=\infty$ on the thick vertical bonds and $\beta=0$ on other vertical bonds. The length of the thick vertical bonds is $2^{p_{1}}-1$ if $x_{1}=2^{p_{1}}+2^{p_{2}}+\ldots$ $\left(p_{1}<p_{2}<\ldots\right)$, and for $x_{1}=0$, all $\beta$ are set $\infty$ on the vertical bonds. The hierarchical lattice (corresponding to $r=2$ and $D=2$ ) of Kadanoff type is obtained by replacing $2^{p_{1}}-1$ by $2^{p_{1}+1}-1$.

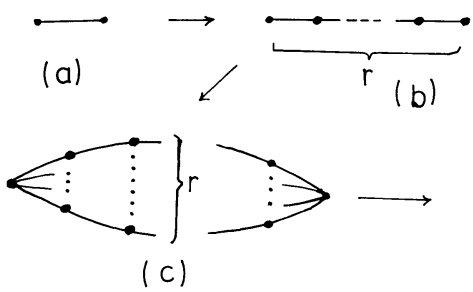

Fig. 2-M. a A unit bond is prepared. b A string of length $r$ is made from unit bonds. $\mathbf{c}$ A block bond is made from strings made in $\mathbf{b}$ with their terminal points identified. Iterate these steps regarding the block bond as a unit bond in thd next distance scale (e.g. $r$ ) 


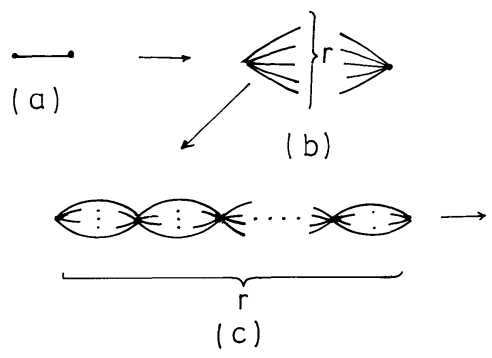

Fig. 2-K. a A unit bond is prepared. $\mathbf{b} r$ unit bonds are combined with their terminal points $\left(u_{i}\right.$ and $u_{i-1}$ ) identified. $\mathbf{c}$ A block bond is made from "bubbles" made in $\mathbf{b}$. Iterate these steps regarding the block bond as a unit bond in the next distance scale (e.g. $r$ )

These systems obviously satisfy the following recursion relations:

(1) Migdal type:

$$
g^{(n+1)}\left(\left(s_{1}, s_{2}\right)\right)=\frac{1}{\mathscr{N}}\left[\int \prod_{i=1}^{r} g^{(n)}\left(\left(u_{i-1}, u_{i}\right)\right) \prod_{i=1}^{r-1} d u_{i}\right]^{r},
$$

(2) Kadanoff type:

$$
g^{(n+1)}\left(\left(s_{1}, s_{2}\right)\right)=\frac{1}{\mathscr{N}} \int\left[\prod_{i=1}^{r} g^{(n)}\left(\left(u_{i-1}, u_{i}\right)\right)\right]^{r} \prod_{i=1}^{r-1} d u_{i},
$$

where $u_{0}=s_{1}, u_{r}=s_{2}, u_{i} \in S^{2}$ and $d u$ denotes the normalized measure on the unit sphere $S^{2} . r$ is a positive integer larger than or equal to 2 , and $\mathscr{N}$ is the normalization constant chosen so that $g^{(n+1)}(1)=1$. Of course, we start with $g^{(0)}\left(\left(s_{1}, s_{2}\right),=\exp \left[\beta\left(\left(s_{1}, s_{2}\right)-1\right)\right]\right.$.

Our discussions are easily extended to the $N$-vector model case in which $S^{2}$ is replaced by $S^{N-1}$. Moreover, we set $r=2$ in Eqs. (1a) and (1b), which somewhat simplifies our arguments. The general $r$ case is easily discussed and our main conclusion does not change. These checks are left to the reader as easy exercises. (See also $[9,11,16]$ on these points.)

Let $e={ }^{t}(1,0,0) \in S^{2}$. Using the fact that $S^{2}$ is a homogeneous space of $\mathrm{SO}(3)$, one can rewrite (1a) and (1b) with $r=2$ as follows:

Migdal type:

$$
g^{(n+1)}(v)=\frac{1}{\mathscr{N}}\left[\int g^{(n)}\left(u^{-1} v\right) g^{(n)}(u) d u\right]^{2}
$$

Kadanoff type:

$$
g^{(n+1)}(v)=\frac{1}{\mathscr{N}} \int\left[g^{(n)}\left(u^{-1} v\right) g^{(n)}(u)\right]^{2} d u,
$$

where $g^{(n)}(v)$ is redefined by $g^{(n)}((e, v e))$. Here $v, u \in \mathrm{SO}(3)$ and $d u$ means the normalized Haar measure on $\mathrm{SO}(3)$. 


\section{Proposition 1.}

$$
1=g^{(n)}(1) \geqq g^{(n)}(v)=g^{(n)}\left(v^{-1}\right) \geqq 0 .
$$

(2) $g^{(n)}(v)$ is of positive type:

$$
\sum_{i, j} \bar{z}_{i} z_{j} g^{(n)}\left(v_{i} v_{j}^{-1}\right) \geqq 0 .
$$

(3) Let $\left\{\lambda_{i}\right\}_{i=1}^{3}$ be $3 \times 3$ traceless real skew-symmetric matrices normalized so that $\left\|\lambda_{i}\right\|=1$. Define $\tau(z)=\exp \left[\sum_{i=1}^{3} z_{i} \lambda_{i}\right]$. Then for any $v, \tilde{v} \in \operatorname{SO}(3), g^{(n)}(v \tau(z) \tilde{v})$ is analytic in $D_{2^{n} \ell}=\left\{\{z\} \in C^{3} ;\left|\operatorname{Im} z_{i}\right| \leqq 2^{n} \ell\right\}$, where $\ell$ is a strictly positive constant which may depend on $\beta$. Moreover, the bound

$$
\left|g^{(n)}(v \tau(z) \tilde{v})\right| \leqq g^{(n)}(v \tau(\operatorname{Re} z) \tilde{v}) \exp \left[\frac{C \beta}{2} \sum_{1}^{3}\left(\operatorname{Im} z_{i}\right)^{2}\right]
$$

holds uniformly in $D_{2^{n} \ell}$, where $C$ is a positive constant (chosen to be independent of $\beta$ ).

Proof. (1) $1 \geqq g^{(n)}(v)$ is trivial by Hölder's inequality. Moreover, since $(e, v e) \in R$, $(e, v e)=(v e, e)=\left(e, v^{*} e\right)=\left(e, v^{-1} e\right)$. Thus $g^{(0)}(v)=g^{(0)}\left(v^{-1}\right)$. For general $n, g^{(n)}\left(v^{-1}\right)$ $=(\mathscr{N})^{-1}\left[\int g^{(n-1)}\left(u^{-1} v^{-1}\right) g^{(n-1)}(u) d u\right]^{2}$, then set $u \rightarrow v^{-1} u$ to find $g^{(n)}\left(v^{-1}\right)=g^{(n)}(v)$. This is obviously also the case for the Kadanoff type recursion formulas.

(2) $\sum_{i, j} \bar{z}_{i} z_{j}\left(e, v_{i}^{t} v_{j} e\right)=\sum_{\ell} \sum_{i, j}\left(\bar{z}_{i}\left(v_{i}\right)_{1 \ell} z_{j}\left(v_{j}\right)_{1 \ell}\right) \geqq 0$. So $(e, v e)$ is of positive type. Then so is $\exp [\beta((e, v e)-1)]$ if $\beta \geqq 0$. Functions of positive type form a positive multiplicative cone and are closed under convolution (Bochner's theorem).

(3) If $g^{(n-1)}$ obeys the bound (5), so does $g^{(n)}$ in $D_{2^{n} \ell}$ because

$$
\begin{aligned}
g^{(n)}(v \tau(z) \tilde{v}) & =(\mathscr{N})^{-1}\left[\int g^{(n-1)}\left(u^{-1} v \tau(z) \tilde{v}\right) g^{(n-1)}(u) d u\right]^{2} \\
& =(\mathscr{N})^{-1}\left[\int g^{(n-1)}\left(u^{-1} \tau\left(\frac{z}{2}\right) \tilde{v}\right) g^{(n-1)}\left(v \tau\left(\frac{z}{2}\right) u\right) d u\right]^{2}
\end{aligned}
$$

So it suffices to prove the assertion for $g^{(0)}(v \tau(z) \tilde{v})=\exp [\beta((e, v \tau(z) \tilde{v} e)-1)]$. Let $A=\sum_{1}^{3}\left(\operatorname{Re} z_{i}\right) \lambda_{i}$ and $B=\sum_{1}^{3}\left(\operatorname{Im} z_{i}\right) \lambda_{i}$ (both are real skew symmetric) so that

$$
\tau(z)=e^{A+i B}=e^{A}+\sum_{n=1}^{\infty}(i)^{n} \int_{t_{i} \geqq 0} \delta\left(\sum t_{i}-1\right) e^{t_{1} A} B e^{t_{2} A} B \ldots B e^{t_{n+1} A} \prod d t_{i} .
$$

Here $e^{t A} \in \operatorname{SO}(3)$, and thus $\left\|e^{t_{i} A}\right\|=1$. Therefore,

$$
\|\operatorname{Re} \tau(z)-\tau(\operatorname{Re} z)\| \leqq \cosh \|B\|-1 \leqq \frac{1}{2}\left(1+O\left(\|B\|^{2}\right)\right)\|B\|^{2}
$$

if $\|B\|$ is small enough. Note that $\|B\|^{2} \leqq$ const $\sum_{1}^{3}\left(\operatorname{Im} z_{i}\right)^{2}$. Since the imaginary part of $\tau(z)$ does not appear in $\left|g^{(0)}(v \tau(z) \tilde{v})\right|$, this completes the proof. This argument is directly applied for the recursion formulas of Kadanoff type. Q.E.D. 
For concreteness, one may take

$$
\lambda_{1}=\left(\begin{array}{rrr}
0 & 1 & 0 \\
-1 & 0 & 0 \\
0 & 0 & 0
\end{array}\right), \quad \lambda_{2}=\left(\begin{array}{rrr}
0 & 0 & 1 \\
0 & 0 & 0 \\
-1 & 0 & 0
\end{array}\right), \quad \lambda_{3}=\left(\begin{array}{rrr}
0 & 0 & 0 \\
0 & 0 & 1 \\
0 & -1 & 0
\end{array}\right)
$$

Moreover, remark that $\operatorname{spec}(v)=\left\{e^{i \theta}, e^{-i \theta}, 1\right\}$ for any $v \in \operatorname{SO}(3)$.

Proposition 2. (1) If $\operatorname{spec}(v)=\left\{e^{i \theta}, e^{-i \theta}, 1\right\}$, then

$$
g^{(n)}(v) \geqq \exp \left[-\frac{\beta}{2}[\theta]_{2 \pi}^{2}\right],
$$

where $[\theta]_{2 \pi} \in[-\pi, \pi)$ and $[\theta]_{2 \pi}=\theta \bmod 2 \pi$.

(2) For $\tau(\theta)=\exp \left(\theta \lambda_{1}\right)$,

$$
\frac{1}{k !}\left|\frac{\partial^{k}}{\partial \theta^{k}} g^{(n)}(v \tau(\theta) \tilde{v})\right| \leqq \operatorname{const} \beta^{k / 2}
$$

uniformly in $v, \tilde{v} \in \mathrm{SO}(3)$ and $n \geqq n_{0}=n_{0}(\beta)$.

Proof. (1) By suitable $u \in \mathrm{SO}(3)$, one can represent $v \in \mathrm{SO}(3)$ as $u \tau(\theta) u^{-1}$, where $\tau(\theta)$ $=\exp \left(\theta \lambda_{1}\right)$. By complexifying $\theta$ and denoting it by $z=\theta+i z_{i}\left(z_{i} \in R\right)$, we define

$$
G(z)=g^{(n)}\left(u \tau(z) u^{-1}\right) \exp \left[\frac{\beta}{2}(1+\varepsilon) z^{2}\right]
$$

in the region $D_{\varepsilon} \subset D_{2^{n} \ell}$, where $\varepsilon$ is small enough. So $G(0)=1$ and

$$
|G(z)| \leqq G(\operatorname{Re} z) \exp \left[-\frac{\beta}{2}\left(\varepsilon-O\left(\varepsilon^{2}\right)\right)(\operatorname{Im} z)^{2}\right],
$$

where we have used

$$
\left|g^{(n)}\left(u \tau(z) u^{-1}\right)\right| \leqq g^{(n)}\left(u \tau(\theta) u^{-1}\right) \exp \left[\frac{\beta}{2}\left(1+O\left((\operatorname{Im} z)^{2}\right)(\operatorname{Im} z)^{2}\right]\right.
$$

which is obtained by setting $B=(\operatorname{Im} z) \lambda_{1}$ and $\|B\|=|\operatorname{Im} z|$ in Ineq. (7) and next applying induction using (6). Note that $O\left((\operatorname{Im} z)^{2}\right)$ can be replaced by $O\left(\left(\operatorname{Im} z / 2^{n}\right)^{2}\right)$. $|G(z)|$ decreases as $|\operatorname{Im} z|$ increases, and then the maximal principle of analytic functions implies $G(\theta) \geqq G(0)=1$, which is Ineq. (9).

(2) By Cauchy's formula, the left-hand side of Ineq. (10) is represented as

$$
\left|\oint_{|z|=R} g^{(n)}(v \tau(\theta+z) \tilde{v}) z^{-k-1} d z\right| .
$$

Set $R=\beta^{-1 / 2}$ and use Ineq. (5). Here $n \geqq n_{0}(\beta)$, where $2^{n_{0}} \ell>\beta^{-1 / 2}$. Q.E.D.

Remarks 1. (1) In the case of lattice gauge theories, $\left\{g^{(n)}(v)\right\}$ are class functions of $G$ $[=\mathrm{SU}(N), \mathrm{SO}(N)$, etc. $]$ within the Migdal-Kadanoff approximations, which is a consequence of the Gauge invariance. In the present case, however, this cannot be satisfied and thus the proof is slightly different from that in [11].

(2) For the $N$-vector model, it is enough to consider the group $\operatorname{SO}(N)$. Thus the extension of our proof to that case is straightforward. 


\section{Proof of the Main Theorem}

Now we show that these recursion formulas drive $g^{(0)}(v)=\exp [\beta((e, v e)-1)]$ into the high temperature region. In other words we show that there exist no fixed points in the recursion formulas except for the trivial $g^{(\infty)}=1$. To do this, we define $\beta_{v, \tilde{v}}^{(n)}(a)$ by

$$
\left|g^{(n)}(v \tau(i a) \tilde{v})\right|=g^{(n)}(v \tilde{v}) \exp \left[\frac{a^{2}}{2} \beta_{v, \tilde{v}}^{(n)}(a)\right]
$$

Then

$$
\beta_{v, \tilde{v}}^{(n)} \equiv \lim _{a \rightarrow 0} \beta_{v, \tilde{v}}^{(n)}(a)=-\left.\frac{\partial^{2}}{\partial \theta^{2}} \ln g^{(n)}(v \tau(\theta) \tilde{v})\right|_{0}
$$

and we define

$$
\beta^{(n)}=\max _{v, \tilde{v}} \beta_{v, \tilde{v}}^{(n)} \geqq 0 .
$$

Because of the bounds (9) and (10), $\beta_{v, \tilde{v}}^{(n)}(a)$ is real analytic in $a$ such that $|a| \ll \beta^{-1 / 2}$, uniformly in $v, \tilde{v} \in \mathrm{SO}(3)$ and $n \geqq n_{0}(\beta)$.

Proposition 3. The following inequality holds uniformly in $n \geqq n_{0}$ :

$$
\left|\beta_{v^{\prime}, \tilde{v}^{\prime}}^{(n)}-\beta_{v, \tilde{v}}^{(n)}\right| \leqq \operatorname{const}\left(\left\|v-v^{\prime}\right\|+\left\|\tilde{v}-\tilde{v}^{\prime}\right\|\right) .
$$

Proof. We already know that $\left|\beta_{v, \tilde{v}}^{(n)}\right|$ is bounded. [To confirm this, apply Proposition 2 to Eq. (14).] So if $\left\|v-v^{\prime}\right\|+\left\|\tilde{v}-\tilde{v}^{\prime}\right\| \geqq \varepsilon>0$, then the proposition is trivial by choosing a large constant in the right-hand side of Ineq. (16). So we assume $v^{\prime}=v v_{0}, \tilde{v}^{\prime}=\tilde{v}_{0} \tilde{v}$ with $\left\|v_{0}-1\right\|<\varepsilon$ and $\left\|\tilde{v}_{0}-1\right\|<\varepsilon$. Here $\varepsilon$ is small enough. Then we may represent $v_{0}=\exp \left[\sum_{1}^{3} \theta_{i} \lambda_{i}\right], \quad \tilde{v}_{0}=\exp \left[\sum_{1}^{3} \varphi_{i} \lambda_{i}\right]$. Of course, $\tau(\theta)=\exp \left[\theta \lambda_{1}\right]$. We can assume $\left|\theta_{i}\right| \leqq$ const $\varepsilon,\left|\varphi_{i}\right| \leqq$ const $\varepsilon$, and $|\theta| \leqq$ const $\varepsilon$. Thus by the Baker-Campbell-Hausdorff formula,

$$
v_{0} \tau \tilde{v}_{0}=\exp \left[\sum_{1}^{3} A_{i} \lambda_{i}\right],
$$

where $A_{1}=\theta+\left(\theta_{1}+\varphi_{1}\right)+O\left(\varepsilon^{2}\right), A_{2}=\left(\theta_{2}+\varphi_{2}\right)+O\left(\varepsilon^{2}\right)$, and $A_{3}=\theta_{3}+\varphi_{3}+O\left(\varepsilon^{2}\right)$ are analytic functions of $\theta,\left\{\theta_{i}\right\}_{1}^{3}$ and $\left\{\varphi_{i}\right\}_{1}^{3}$ in a neighborhood of the origin. Since

$$
\beta_{v^{\prime}, \tilde{v}^{\prime}}^{(n)}(0)=-\left[g^{(n)}\left(v^{\prime}, \tilde{v}^{\prime}\right)^{\prime \prime} g^{(n)}\left(v^{\prime} \tilde{v}^{\prime}\right)-\left(g^{(n)}\left(v^{\prime}, \tilde{v}\right)^{\prime}\right)^{2}\right]\left(g^{(n)}\left(v^{\prime} \tilde{v}^{\prime}\right)\right)^{-2},
$$

where $g^{(n)}\left(v^{\prime}, \tilde{v}^{\prime}\right)^{\prime}=\left.\frac{\partial}{\partial \theta} g^{(n)}\left(v^{\prime} \tau(\theta) \hat{v}^{\prime}\right)\right|_{0}$, etc., and since $g^{(n)}(v) \geqq$ const $>0$, it is enough to show that $g^{(n)}\left(v v_{0} \tilde{v}_{0} \tilde{v}\right), g^{(n)}\left(v v_{0}, \tilde{v}_{0} \tilde{v}\right)^{\prime}$, and $g^{(n)}\left(v v_{0}, \tilde{v}_{0} \tilde{v}\right)^{\prime \prime}$ are uniformly continuous in $\left\{\theta_{i}, \varphi_{i}\right\}_{1}^{3}$ such that $\left|\theta_{i}\right|,\left|\varphi_{i}\right| \leqq$ const $\varepsilon$, uniformly in $v$ and $\tilde{v}$. To do so, we remark that $g^{(n)}\left(v \exp \left[\sum A_{i} \lambda_{i}\right] \tilde{v}\right)=(2 \pi i)^{-3} \oint g^{(n)}\left(v \exp \left[\sum z_{i} \lambda_{i}\right] \tilde{v}\right) \prod\left(z_{i}-A_{i}\right)^{-1} d z_{i}$, where $\left|z_{i}\right|=\beta^{-1 / 2}$. Since $A_{i}$ 's are analytic functions such that $A_{i} \rightarrow 0$ as $\theta, \theta_{k}, \varphi_{k} \rightarrow 0$, the conclusion follows immediately by choosing small $\varepsilon\left(\varepsilon \ll \beta^{-1 / 2}\right)$. Q.E.D. 
Now we are going to prove our first main theorem. Since the proof is completely same, we discuss the recursion formulas of Migdal type only.

$$
g^{(n+1)}\left(v \tau(i a) v^{\prime}\right)=\frac{1}{\mathscr{N}}\left[\int g^{(n)}\left(u^{-1} \tau\left(\frac{i a}{2}\right) v^{\prime}\right) g^{(n)}\left(v \tau\left(\frac{i a}{2}\right) u\right) d u\right]^{2} .
$$

So taking the absolute values of both sides and choosing $a$ small, we have:

$$
\begin{aligned}
& g^{(n+1)}\left(v v^{\prime}\right)\left(1+\frac{a^{2}}{2} \beta_{v, v^{\prime}}^{(n+1)}+O\left(a^{4}\right)\right) \\
& \quad \leqq \frac{1}{\mathscr{N}}\left[\int g^{(n)}\left(u^{-1} v^{\prime}\right) g^{(n)}(v u)\left[1+\frac{a^{2}}{8}\left(\beta_{u^{-1}, v^{\prime}}^{(n)}+\beta_{v, u}^{(n)}\right)+O\left(a^{4}\right)\right] d u\right]^{2},
\end{aligned}
$$

where by definition, $\mathscr{N}=\left[\int g^{(n)}(u)^{2} d u\right]^{2}$. Then

$$
0 \leqq \lim _{a \rightarrow 0} a^{-2} \quad \text { [RHS of Ineq. (20) - LHS of Ineq. (20)], }
$$

which yields

$$
\beta_{v, v^{\prime}}^{(n+1)} \leqq \frac{\int g^{(n)}\left(u^{-1} v^{\prime}\right) g^{(n)}(v u)\left[\beta_{u^{-1}, v^{\prime}}^{(n)}+\beta_{v, u}^{(n)}\right] d u}{2 \int g^{(n)}\left(u^{-1} v^{\prime}\right) g^{(n)}(v u) d u} .
$$
We choose $v, v^{\prime}$ such that $\max _{v_{1}, v_{2}} \beta_{v_{1}, v_{2}}^{(n+1)}=\beta_{v, v^{\prime}}^{(n+1)}$. Such a pair $\left(v, v^{\prime}\right)$ may not be unique.
So

$$
\beta^{(n+1)}-\beta^{(n)} \leqq-\frac{\int g^{(n)}\left(u^{-1} v^{\prime}\right) g^{(n)}(v u)\left[\beta^{(n)}-\Delta^{(n)}\left(u ; v, v^{\prime}\right)\right] d u}{\int g^{(n)}\left(u^{-1} v^{\prime}\right) g^{(n)}(v u) d u},
$$

where $\Delta^{(n)}\left(u ; v, v^{\prime}\right)=\frac{1}{2}\left(\beta_{u^{-1}, v^{\prime}}^{(n)}+\beta_{v, u}^{(n)}\right) \leqq \beta^{(n)}$, which means that $\left\{\beta^{(n)}\right\}$ are monotone decreasing in $n$. Note that $\beta_{v, \tau(\theta) v^{\prime}}^{(n)}$ is a periodic function of $\theta$ such that $\int_{0}^{2 \pi} \beta_{v, \tau(\theta) v^{\prime}}^{(n)} d \theta=0$. So unless $g^{(n)} \equiv 1, \beta^{(n)}>0$ and $\min \beta_{v, v^{\prime}}^{(n)}<0$. Moreover, there exists $u \in \mathrm{SO}(3)$ for any $v^{\prime} \in \mathrm{SO}(3)$ such that $\beta_{u^{-1}, v^{\prime}}^{(n)}<0$.

Therefore, if $\lim \beta^{(n)}=\beta_{c}>0$, then Ineq. (3), the bound (9), and Proposition 3 imply that there must be a strictly positive constant $\kappa$ such that

$$
\beta^{(n+1)}-\beta^{(n)} \leqq-\kappa
$$

So $\beta^{(n)} \rightarrow-\infty$, which is a contradiction.

\section{Theorem 4.}

$$
\lim \beta^{(n)}=0
$$

(2) $\lim g^{(n)}(v)=1$, uniformly in $v \in \mathrm{SO}(3)$.

Proof. (1) This has been proved.

(2) Let $v=u \tau(\theta) u^{-1}$ by suitable $u \in \operatorname{SO}(3)$. Because of Proposition $1(1), g^{(n)}(v)$ is even and analytic in $\theta$. Then

$$
\ln g^{(n)}(v)=\int_{0}^{\theta} d s\left[\ln g^{(n)}\left(u \tau(s) u^{-1}\right)\right]^{\prime}=\int_{0}^{\theta} d s \int_{0}^{s} d t\left[\ln g^{(n)}\left(u \tau(t) u^{-1}\right)\right]^{\prime \prime} \geqq-\frac{\beta^{(n)}}{2}[\theta]_{2 \pi}^{2},
$$

where we have used $\ln g^{(n)}(1)=0,\left[\ln g^{(n)}\left(u \tau(s) u^{-1}\right)\right]_{s=0}^{\prime}=0$ and the definition of $\beta^{(n)}$. Thus $1 \geqq g^{(n)}(v) \geqq \exp \left[-\left(\beta^{(n)} / 2\right)[\theta]_{2 \pi}^{2}\right]$. Q.E.D. 


\section{Correlation Length}

Since $g^{(n)}(v)$ is a continuous function on $G=\mathrm{SO}(3)$ of positive type, we have

$$
g^{(n)}(v)=C_{0}^{(n)}+\sum_{\sigma \neq 0} \sum_{i, j} C_{\sigma, i j}^{(n)}\left(U_{\sigma}\right)_{i j}(v),
$$

where $U_{\sigma}$ is the representation of $G=\operatorname{SO}(3)$ belonging to the character $\sigma$ and

$$
C_{\sigma, i j}^{(n)} \geqq 0, \quad C_{0}^{(n)}+\sum_{\sigma \neq 0} \sum_{i} C_{\sigma, i i}^{(n)}=1 .
$$

Here the second equation comes from the normalization condition of $g^{(n)}$.

Choosing $n_{0}=n_{0}(\beta)$ large, we can assume

$$
1 \geqq g^{\left(n_{0}\right)}(v) \geqq 1-\varepsilon_{n_{0}},
$$

where $1 \gg \varepsilon_{n_{0}}>0$. Set

$$
g^{\left(n_{0}\right)}(v)=C_{0}^{\left(n_{0}\right)}+\delta g^{\left(n_{0}\right)}(v)
$$

Then

$$
\begin{gathered}
1 \geqq C_{0}^{\left(n_{0}\right)}=\int g^{\left(n_{0}\right)}(v) d v \geqq 1-\varepsilon_{n_{0}}, \\
\left|\delta g^{\left(n_{0}\right)}(v)\right|=\left|g^{\left(n_{0}\right)}(v)-\int g^{\left(n_{0}\right)}(u) d u\right| \leqq \varepsilon_{n_{0}},
\end{gathered}
$$

which imply

$$
\begin{aligned}
& \varepsilon_{n_{0}+1} \equiv \max \left(1-g^{\left(n_{0}+1\right)}(v)\right)=\max \left[1-\frac{\left[\left(C_{0}^{\left(n_{0}\right)}\right)^{2}+\left(\delta g^{\left(n_{0}\right)} * \delta g^{\left(n_{0}\right)}\right)(v)\right]^{2}}{\left[\left(C_{0}^{\left(n_{0}\right)}\right)^{2}+\left\|\delta g^{\left(n_{0}\right)}\right\|^{2}\right]^{2}}\right] \\
& \leqq 1-\left[\frac{\left(C_{0}^{\left(n_{0}\right)}\right)^{2}-\left(\varepsilon_{n_{0}}\right)^{2}}{\left(C_{0}^{\left(n_{0}\right)}\right)^{2}+\left(\varepsilon_{n_{0}}\right)^{2}}\right]^{2}=1-\left[1-\frac{2 \varepsilon_{n_{0}}^{2}}{\left(C_{0}^{\left(n_{0}\right)}\right)^{2}+\left(\varepsilon_{n_{0}}\right)^{2}}\right]^{2} \leqq \frac{4 \varepsilon_{n_{0}}^{2}}{\left(C_{0}^{\left(n_{0}\right)}\right)^{2}+\varepsilon_{n_{0}}^{2}} \leqq \kappa \varepsilon_{n_{0}}^{2}
\end{aligned}
$$

if $\varepsilon_{n_{0}}$ is small enough, where $*$ denotes the convolutive product defined in the obvious way and $\kappa$ is a positive constant which can be chosen less than 5 by choosing $n_{0}$ large. Then $\kappa \varepsilon_{n_{0}+1} \leqq\left(\kappa \varepsilon_{n_{0}}\right)^{2}$, and thus we have:

$$
\begin{gathered}
\varepsilon_{n} \leqq(\kappa)^{-1}\left(\kappa \varepsilon_{n_{0}}\right)^{2^{n-n_{0}}}=(\kappa)^{-1} \exp \left[-\tilde{L} 2^{n-n_{0}}\right], \\
\tilde{L}=-\ln \left(\kappa \varepsilon_{n_{0}}\right)>0,
\end{gathered}
$$

where $n \geqq n_{0}$ and $n_{0}=n_{0}(\beta)$ has been chosen so that $\kappa \varepsilon_{n_{0}}<1$.

Theorem 5. For $n \geqq n_{0}(\beta)$,

$$
0 \leqq 1-g^{(n)}(v) \leqq C_{1} \exp \left[-C_{2} 2^{n-n_{0}}\right],
$$

where $C_{1}$ and $C_{2}$ are strictly positive constants.

Remarks 2. (1) From (29a), it is seen that $\varepsilon_{n_{0}} \leqq 1 / 6$ is enough for our purpose. In fact, $4 \varepsilon_{n_{0}}\left[\left(1-\varepsilon_{n_{0}}\right)^{2}+\varepsilon_{n_{0}}^{2}\right]^{-1} \leqq 12 / 13$ is less than 1 in this case.

(2) The proof of Theorem 5 is a kind of high-temperature expansion applied to the present hierarchical system.

So far we have not used the fact that $\left\{g^{(n)}(v)\right\}$ are functions of positive type which ensure the expansions (25) together with (26). Let $\Lambda=\left[0,2^{N}\right] \times\left[0,2^{N}-1\right] \cap Z^{2}$, and let $s=s_{x}$ and $s^{\prime}=s_{x^{\prime}}$ where $x=\left(x_{1}, x_{2}\right)$, 
$x^{\prime}=\left(x_{1}+2^{n}, x_{2}\right)$ and $x_{1}=k 2^{n}$ for some $k \in Z, k \geqq 0$. These two points are connected after $n$ iterations of the recursion formulas on the hierarchical lattice, see Fig. 1. Let

$$
h^{(n+1)}(v)=\mathscr{N}_{n+1}^{-1}\left[\int g^{(n)}\left(u^{-1} v\right) g^{(n)}(u)(u)_{11} d u\right]\left[\int g^{(n)}\left(u^{-1} v\right) g^{(n)}(u) d u\right],
$$

where $u \in \mathrm{SO}(3)$ is introduced so that $s_{x} \cdot s_{x^{\prime}}=(e, u e)$ and $\mathscr{N}_{n+1}$ is the normalization constant chosen so that $g^{(n+1)}(1)=1 . h^{(n+1)}$ is also of positive type again by Bochner's theorem and satisfies

$$
\left|h^{(n+1)}(v)\right| \leqq \text { const } \exp \left[-C_{2} 2^{n-n_{0}}\right] \leqq C_{1}^{\prime} \exp \left[-C_{2} 2^{n-n_{0}}\right] g^{(n+1)}(v)
$$

by Theorem 5 and $\int(u)_{11} d u=0$, where $C_{1}^{\prime}$ is a positive constant. Therefore,

$$
h^{(n+\ell+1)}(v)=\mathscr{N}_{n+\ell+1}^{-1}\left[\int h^{(n+\ell)}\left(u^{-1} v\right) g^{(n+\ell)}(u) d u\right]\left[\int g^{(n+\ell)}\left(u^{-1} v\right) g^{(n+\ell)}(u) d u\right]
$$

with $l=0,1, \ldots$ are functions of positive type satisfying the bounds

$$
\left|h^{(n+\ell+1)}(v)\right| \leqq C_{1}^{\prime} \exp \left[-C_{2} 2^{n-n_{0}}\right] g^{(n+\ell+1)}(v) .
$$

Thus we have

$$
\begin{gathered}
0 \leqq\left\langle s s^{\prime}\right\rangle_{\Lambda} \leqq C_{1}^{\prime} \exp \left[-m(\beta) 2^{n}\right], \\
m(\beta)=C_{2} 2^{-n_{0}(\beta)},
\end{gathered}
$$

uniformly in $N$ or in $\Lambda$. This implies that the system has a finite correlation length $\xi(\beta) \leqq m(\beta)^{-1}<\infty$, which also holds for the hierarchical systems of Kadanoff type.

Remark 3. The positivity of $\left\langle s s^{\prime}\right\rangle_{A}$ is a consequence of the positivity of $\left\{g^{(n)}\right\}$ and $\left\{h^{(n)}\right\}$. But this is easily understood by Griffith's first inequality since the system is ferromagnetic. See the caption for Fig. 1.

\section{Remaining Problems and Discussions}

We have shown that the 2D Heisenberg model is always in the massive phase within the Migdal-Kadanoff approximate formulas. Though this is consistent with our conjecture, we must admit that the 2D $X Y$-model does not exhibit the Kosterlitz-Thouless transitions within the Migdal-Kadanoff approximate formulas, because as is easily understood, the present method of analysis can be easily applied for $G=\mathrm{SO}(2)$. (In fact, the proof is much easier in this case [10].) This implies unfortunately that Migdal's and Kadanoff's ideas are not accurate enough to pick up very crucial properties of non-Abelian symmetries which Abelian systems do not have and are supposed to be responsible for asymptotic freedom in short distances in these systems. See $[12,7]$ for a recent study in this direction.

A study of the present system by the rigorous real space renormalization group methods is now under progress, which will be reported in the near future.

\section{References}

1. Bałaban, T.: Renormalization group approach to lattice gauge field theories (I). Harvard University preprint (HUTMP-B189)

Bałaban, T., Jaffe, A.: Constructive gauge theory. Harvard University preprint (HUTMPB191) 1985 Erice lectures, to appear 
2. Bleher, P.M., Major, P.: Renormalization of Dyson's hierarchical vector valued $\phi^{4}$ model at low temperatures. Commun. Math. Phys. 95, 487 (1984)

3. Collet, P., Eckmann, J.: A spin glass model with random couplings. Commun. Math. Phys. 93, 379 (1984)

Collet, P., Eckmann, J., Glaser, V., Martin, A.: Study of the iteration of a mapping associated to a spin glass model. Commun. Math. Phys. 94, 358 (1984)

4. Fröhlich, J., Pfister, C.-E.: On the absence of spontaneous symmetry breaking and of crystalline ordering in two-dimensional systems. Commun. Math. Phys. 81, 277 (1981)

5. Federbush, P.: A phase cell approach to Yang-Mills theory. I. Commun. Math. Phys. 107, 319-329 (1986)

6. Gawedzki, K., Kupiainen, A.: A rigorous block spin approach to massless lattice theories. Commun. Math. Phys. 77, 31 (1980)

Gawedzki, K., Kupiainen, A.: Non-Gaussian fixed points of the block spin transformation. Hierarchical model approximation. Commun. Math. Phys. 89, 191 (1983)

7. Gawedzki, K., Kupiainen, A.: Continuum limit of the hierarchical nonlinear $O(N)$ sigmamodel. Commun. Math. Phys. 106, 533 (1986)

8. Griffiths, R., Kaufman, M.: Spin systems on hierarchical lattices. Introduction and thermodynamic limit. Phys. Rev. 26 B, 5022 (1982)

Griffiths, R., Kaufman, M.: Spin systems on hierarchical lattices. II. Some examples of soluble models. Phys. Rev. 30 B, 244 (1984)

9. Ito, K.R.: Analytic study of Migdal-Kadanoff recursion formulas. Commun. Math. Phys. 95, $247(1984)$

10. Ito, K.R.: Absence of the Kosterlitz-Thouless fixed points in the Migdal-Kadanoff recursion formulas. Phys. Rev. Lett. 54, 2383 (1985)

11. Ito, K.R.: Permanent quark confinement in four-dimensional hierarchical lattice gauge theories of Migdal-Kadanoff type. Phys. Rev. Lett. 55, 558 (1985)

Ito, K.R.: Renormalization group methods on hierarchical lattices. Suppl. Prog. Theor. Phys. (to be published) (1987)

12. Ito, K.R.: Origin of asymptotic freedom in non-Abelian field theories. Phys. Rev. Lett. 58, 439 (1987), and a paper in preparation

13. Kadanoff, L.: Notes on Migdal's recursion formulas. Ann. Phys. 100, 359 (1976)

14. Ma, S.K.: The modern theory of critical phenomena, Chap. 8. Reading, MA: Benjamin 1976

15. Migdal, A.A.: Recursion equations in gauge field theories. JETP 69, 810 (1975)

Migdal, A.A.: Phase transition in gauge and spin-lattice systems. JETP 69, 558 (1976)

16. Müller, V., Schiemann, J.: Convergence of Migdal-Kadanoff iterations in non-abelian lattice gauge models. Commun. Math. Phys. 97, 605 (1985)

17. Müller, V., Schiemann, J.: Continuum limit of a hierarchical SU(2) lattice gauge theory in 4 dimensions. Commun. Math. Phys. (in press)

18. Shlosman, S.B.: Phase transition for two-dimensional models with isotropic short-range interaction and continuous symmetries. Commun. Math. Phys. 71, 207 (1980)

19. Wilson, K.G.: Confinement of quarks. Phys. Rev. D 10, 2445 (1974)

20. Wilson, K.G., Kogut, J.: The renormalization group and $\varepsilon$-expansion. Phys. Rep. 12 C, 75 (1974)

Communicated by J. Fröhlich

Received July 30, 1986; in revised form December 5, 1986

Note added in proof. It may be helpful for the reader to explain how Eqs. $(2 \mathrm{a}, \mathrm{b})$ are obtained from Eqs. $(1 \mathrm{a}, \mathrm{b})$. Let $H=\{h \in \mathrm{SO}(3)=G ; h e=e\}$. Then $H \cong \mathrm{SO}(2)$ and $G / H \cong S^{2}$. Correspondingly the Haar measure $d u$ on $G$ is expressed as $d s d h$, where $d h$ is the Haar measure on $H$ and $d s$ is the measure on $S^{2}$. Since $\left(v_{i} e, u h e\right)=\left(v_{i} e, u e\right)$ where $v_{i} e=s_{i}(i=1,2), u \in G$ and $h \in H$, the Haar measure $d u$ is reduced to $d s$. 